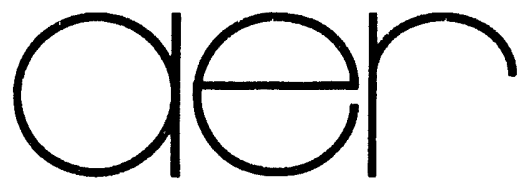

Progress Report No. 3

A Research Program

\title{
on \\ Radiative Transfer Model Development in Support of the ARM Program
}

A research program funded by the

Office of Energy Research

Grant No. DE-FG02-90ER61064

For the period

1 November 1992 - 31 October 1993

Submitted by

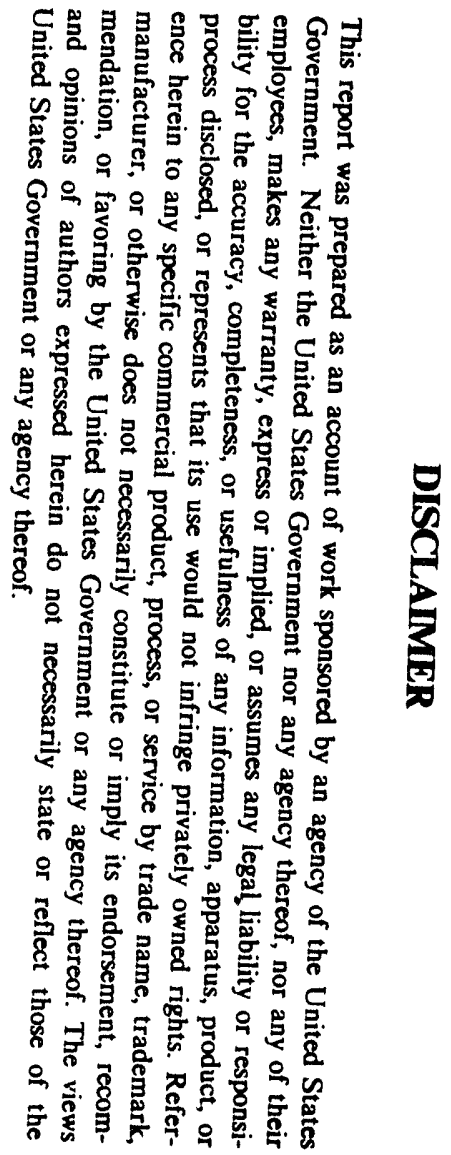

Atmospheric and Environmental Research, Inc. 840 Memorial Drive

Cambridge, MA 02139

1 October 1993

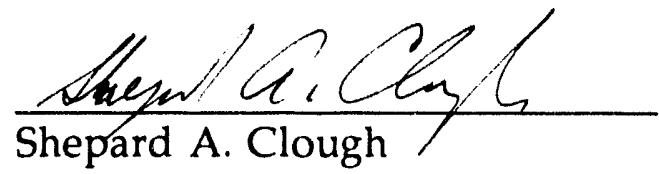

Principal Investigator

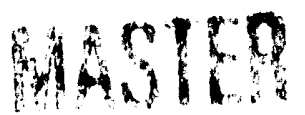




\section{Progress for the Reporting Period}

The objective of this research effort is to develop radiative transfer models that are consistent with ARM spectral radiance measurements for clear and cloudy atmospheres. Our approach is to develop the model physics and related databases with a line-by-line model in the context of available spectral radiance measurements. The line-by-line model then functions as an intermediate standard to both develop and validate rapid radiative transfer models appropriate to GCM applications. A preprint of an extended abstract for the 1994 AMS volume describing a Quality Measurement Experiment using the ARM spectral data is included as an attachment.

\section{Line-by-line Multiple Scattering Code: CHARTS}

CHARTS (Code for High-resolution Atmospheric Radiative Transfer with Scattering), J.-L. Moncet lead investigator, is now fully functional for applications in the thermal infrared. The code is optimized to calculate the spectral radiance and spectral transmittance for a scattering atmosphere with the viewing angle and position specified. These are the anticipated conditions for application of the code to anticipated ARM spectral observations: ground, aircraft or satellite based spectral radiance and transmittance measurements. CHARTS restricts the multiple scattering calculation to the atmospheric regime in which the scatterer is included. A line-by-line radiative transfer model, in this case LBLRTM, is used for the non-scattering atmosphere in the altitude regime above the clouds, providing the spectral dependent boundary condition for the CHARTS calculation. Figure 1 demonstrates the effects on the down welling spectral radiances due to multiple scattering from an optically thin cirrus cloud in the altitude regime from $10-12 \mathrm{~km}$ for a range of cloud water paths. This case (S. Ackerman, 1993) is consistent with downlooking data taken with the HIS instrument (Smith et al., 1983) from $20 \mathrm{~km}$ and with simultaneous data taken with the AERI instrument on the ground as part of the SPECTRE experiment. The spectral scattering parameters have been calculated for ice spheres with a $15 \mathrm{~m}$ mode radius by Ackerman. The cloud, covering the altitude regime from 10-12 km, has a mass path $5 \mathrm{~g} / \mathrm{m}^{2}$. Validations are being performed using these data sets and comparing the results of our model with results from more rapid but less rigorous treatments of the multiple scattering. Extension of the model to solar spectral transmittance measurements is being initiated.

\section{Radiation Models: LBLRTM and RRTM}

Extensive effort has gone into two aspects of the Line-By-Line Radiative Transfer Model (LBLRTM): (1) the application of the model to real time spectral validations for ARM measurements including the implementation of an fft package to model spectral instrument functions and (2) the adaptation of the model to provide the necessary interface 


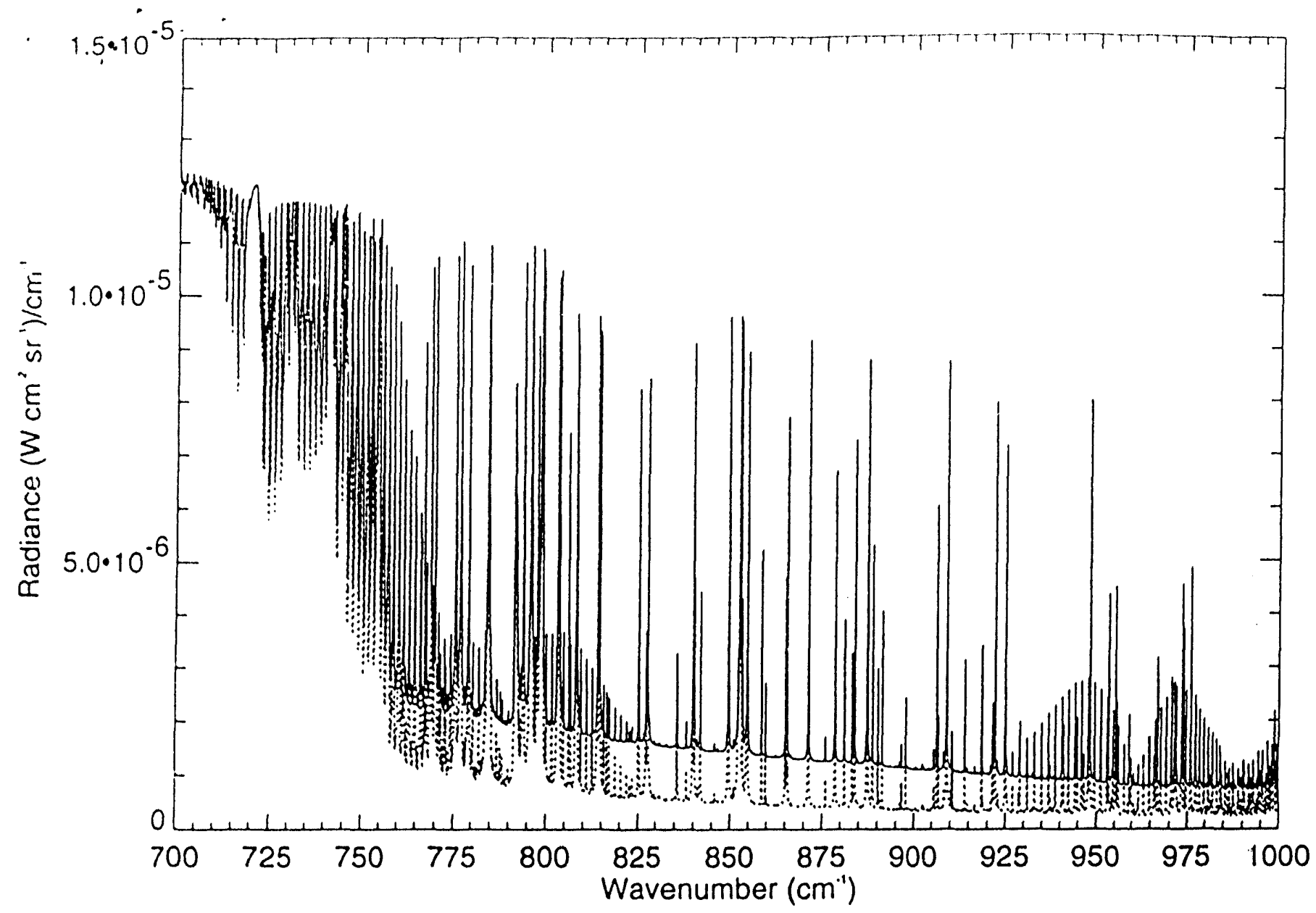

Figure 1a. The down welling spectral radiance at the surface for an atmosphere with a 10$12 \mathrm{~km}$ optically thin cirrus cloud with $15 \mathrm{~m}$ mode radius and mass path of $5 \mathrm{~g} / \mathrm{m}^{2}$ (solid curve) and for a clear atmosphere (dotted curve).

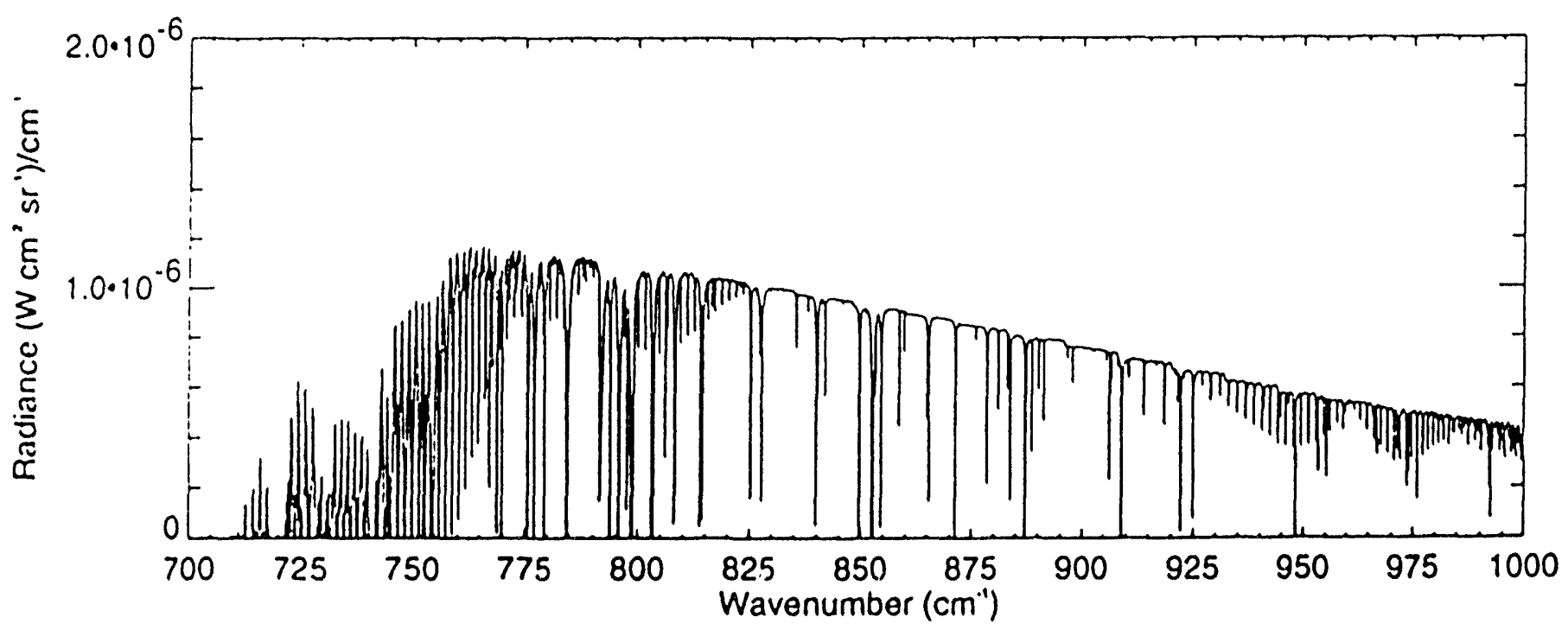

Figure $1 b$. Difference in spectral radiances of figure $1 \mathrm{a}$, cloud - clear. 
with CHARTS. LBLRTM is a vectorizable ine-by-line model based on the FASCODE algorithms and includes the capability to calculate atmospheric fluxes and heating rates. The model has also been adapted to function as the forward model in a etrieval algorithm for the retrieval of atmospheric profiles of temperature and trace gas profiles from high resolution spectral radiance data. LBLRTM has been extensively validated with HIS measurements, e.g. Clough et al. (1992).

In the course of developing a rapid radiative transfer model (RRTM) we have performed spectral band comparisons of cooling/heating rate results from the NASA AMES/Penn. State model [Toon et al., 1989], with line-by-line results obtained from LBLRTM. The results at this stage of the effort indicate that the treatment of the continuum and the k-distributions for the line contribution in this rapid model are not consistent with LBLRTM. At the surface there is a difference of $10 \mathrm{w} / \mathrm{m} 2$ in the net flux for the midlatitude summer atmosphere. Since it is the goal of the present research effort to attain accuracy performance from the rapid model that is consistent with LBLRTM and measurements to be taken at the ARM sites, an initiative is underway to develop a new RRTM that utilizes a continuum formulation and $k$-distributions that provide results as consistent as possible with the line-by-line calculations.

\section{Revised Continuum Model}

The water vapor continuum plays an important role in atmospheric radiative transfer providing increased opacity between spectral lines over the full spectral region from the microwave to the visible. The continuum has a significant influence on atmospheric fluxes and cooling rates. Additionally the continuum is important to the physical solution of the inverse problem, the remote sensing of atmospheric state to retrieve temperature, water vapor, surface properties and other state parameters. There are two components to the continuum: the self-broadened continuum, dependent on the square of the partial pressure of water vapor, and the foreign-broadened continuum, dependent on the product of the water vapor partial pressure and the dry air pressure. As a consequence the self broadened continuum tends to be more important in the lower atmosphere while the foreign broadened continuum tends to be more important in the middle to upper troposphere. A comprehensive continuum modei based on a single line shape for all transitions from the microwave to the visible has provided generally acceptable results (Clough et al., 1989 and Clough et al., 1980; hereafter CKD). The nadir and zenith spectral radiometric measurements with the University of Wisconsin HIS instrument have been particularly useful in establishing the general level of accuracy for the continuum. For most spectral regions the apparent error in the continuum is of the order of $10 \%$ or less. The term 'apparent' is used because it is difficult to unambiguously characterize the atmosphere, particularly with respect to aerosol loading and to sub-visual cloud effects. However, for special conditions of atmospheric 
state, measurements indicate that for specific and very limited spectral regions, errors in the CKD continuum may be significant.

To address this situation and to improve overall accuracy of atmospheric radiance calculations, we have developed an improved but preliminary water vapor continuum model, designated CKD-1. The approach has been to more carefully model the laboratory measurements of Burch and to validate the refined model with up and down looking spectral radiance data. The full data set of Burch and coworkers $(1985,1984,1981)$ has been reanalyzed using a self-consistent approach for the contribution of local lines. In contrast to the earlier model, this reanalysis provides full consistency with the line shape decomposition used for the LBLRTM model. It had been our intention to develop a new water vapor line shape which, when applied to all water vapor lines, would provide agreement with the laboratory and atmospheric measurements. Evidence is mounting that for the accuracy required for ARM radiation measurements and for climate change considerations, a continuum model based either on semi-empirical analytic line shape functions (CKD) or those obtained from collisional theory is not likely to prove adequate. Ma and Tipping using the quasistatic approximation (e.g. 1992 and private communication), have developed a line shape which they have utilized to obtain a self and foreign water vapor continuum. In our judgment their results are not sufficiently accurate to be used directly to describe the continuum; a calculation using the molecular potential of the water molecule would not be expected to be valid to the required level of accuracy.

The continuum absorption coefficient for a homogeneous path, $\mathrm{k}_{\mathrm{c}}$, may be expressed in terms of the self and foreign continuum coefficients, $\tilde{C}_{\mathrm{s}}$ and $\tilde{C}_{\mathrm{f}}$, as

$$
k_{c}=v \tanh (h c v / k T)\left[\left(\rho_{h_{2} o} / \rho_{o}\right) \tilde{C}_{s}(T)+\left(\rho_{\text {air }} / \rho_{o}\right) \tilde{C}_{f}(T)\right]
$$

where $\mathrm{n}$ is the photon wavenumber value, $\mathrm{T}$ is the temperature, $\mathrm{r}_{\mathrm{h} 2 \mathrm{o}}$ is the density of water vapor, $r_{a i r}$ is the density of dry air and $r_{0}$ is the reference density at one atmosphere. Figures $2 \mathrm{a}$ and $2 \mathrm{~b}$ provide the self and foreign continuum coefficients respectively for the longwave region. The symbols represent the measurements of Burch with the local line contribution removed using the LBLRTM local line definition at the indicated temperature. In both the self and foreign cases the CKD continuum does not model the data well at the low wavenumber side of the $\mathrm{n}_{2}$ band in the $1300 \mathrm{~cm}^{-1}$ region. The CKD- 1 continuum has been analytically adjusted to provide improved agreement. A validation of this modification is provided in figure 3. Figure 3 a provides the upwelling spectral radiances measured at $20 \mathrm{~km}$ with the HIS instrument, figure $3 \mathrm{~b}$ provides the spectral residuals with the CKD continuum and figure $3 \mathrm{c}$ provides the spectral residuals with the revised CKD-1 continuum. Significant improvements in the spectral residuals are apparent. The remaining residuals in the $1300 \mathrm{~cm}^{-1}$ region are not attributable to the continuum since removal of the 


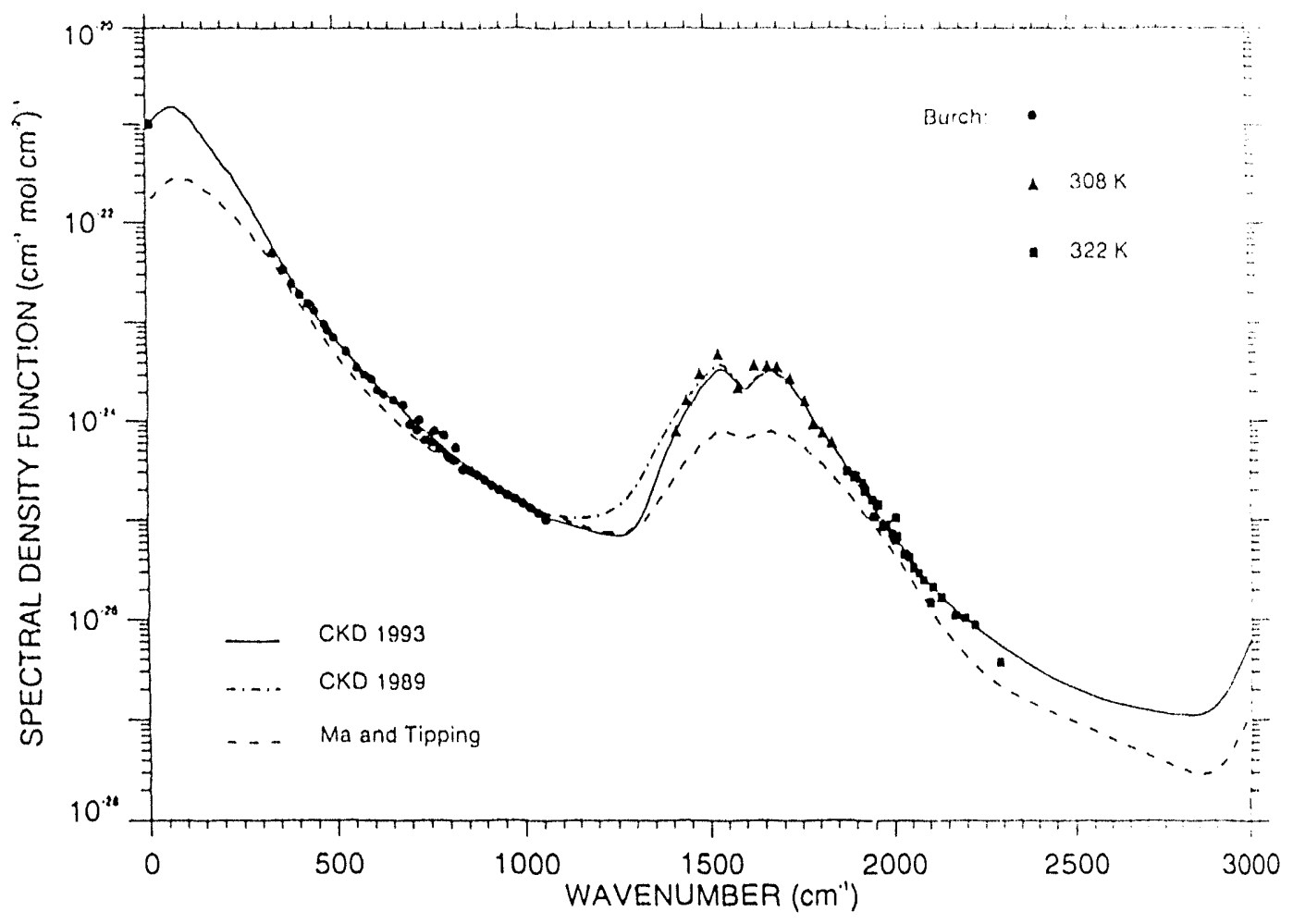

Figure $2 \mathrm{a}$. The water vapur continuum coefficients at $296 \mathrm{~K}$ for the self-broadening case.

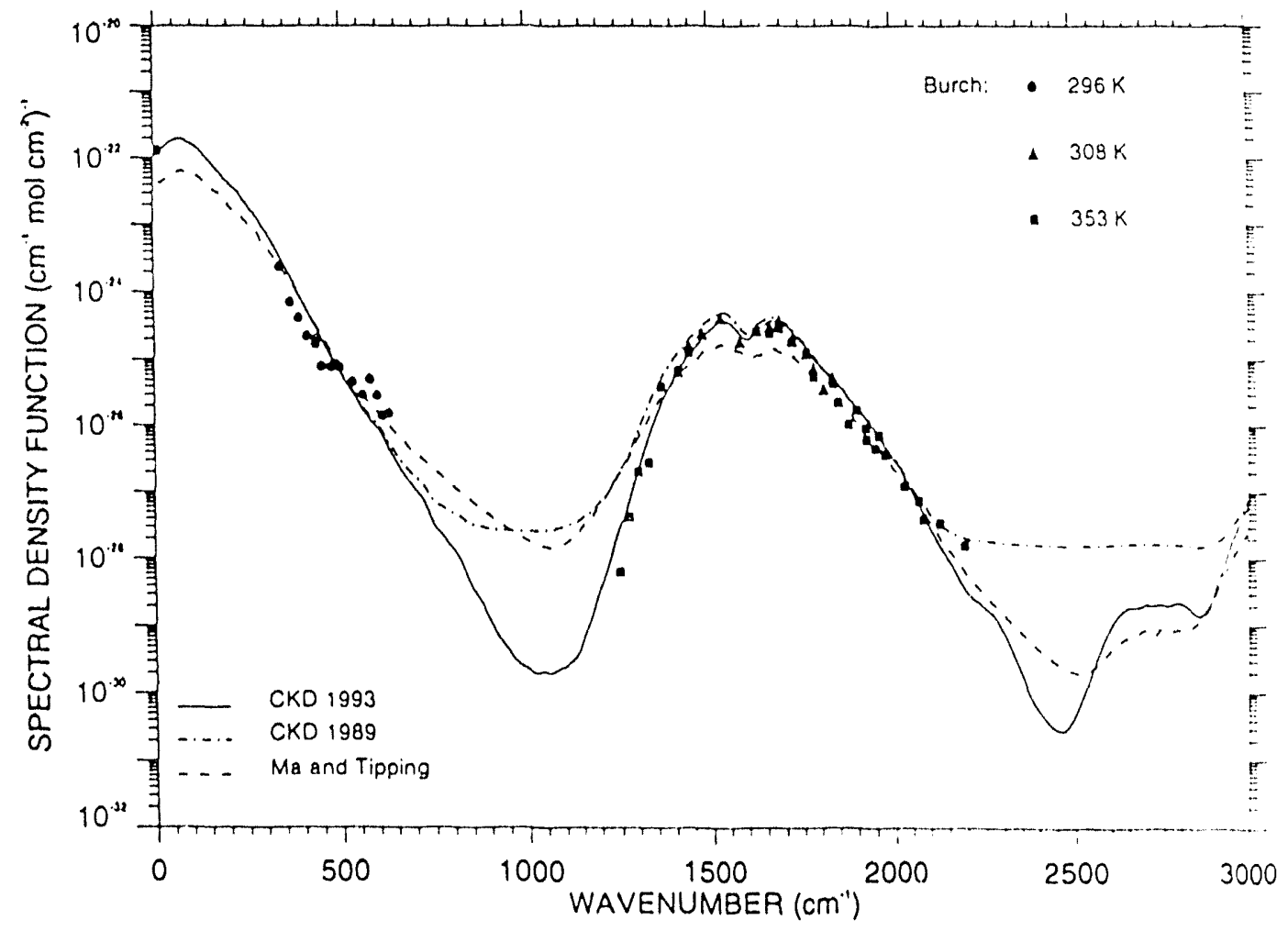

Figure $2 \mathrm{~b}$. The water vapor continuum coefficients at $296 \mathrm{~K}$ for the foreign-broadening case. 
CHANGES TO CONTINUUM

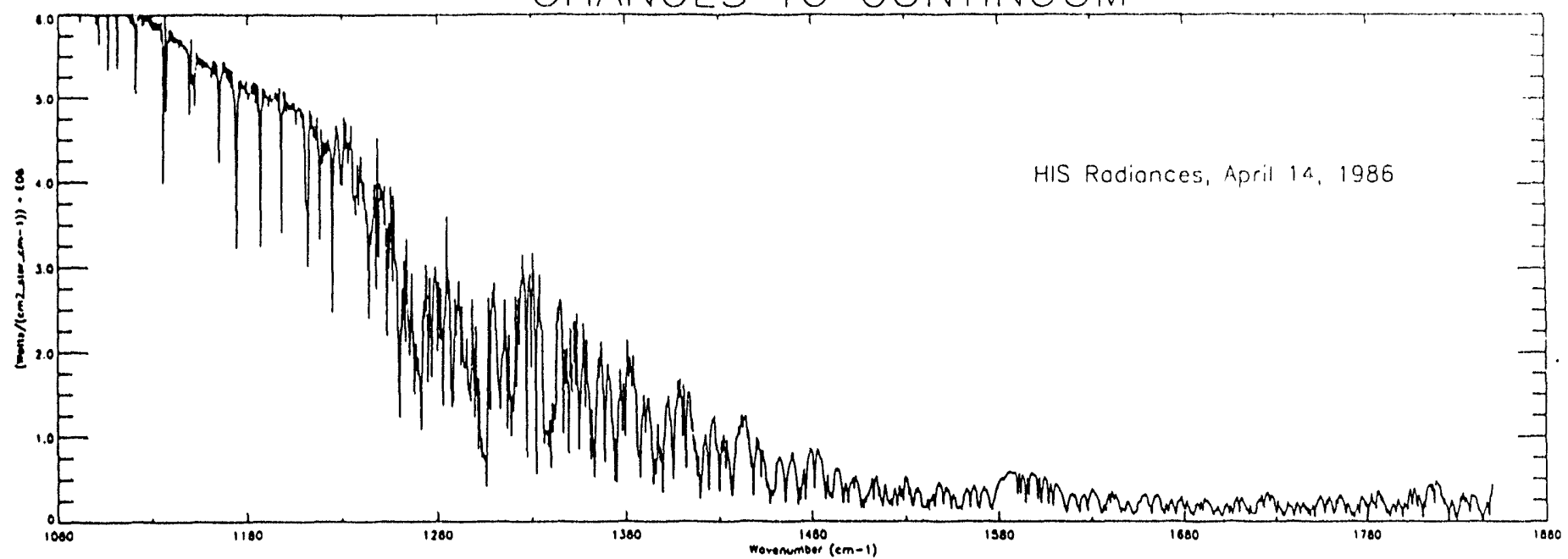

Figure 3a. Spectral radiances from the 14 April 1986 HIS measurement from $19.6 \mathrm{~km}$.

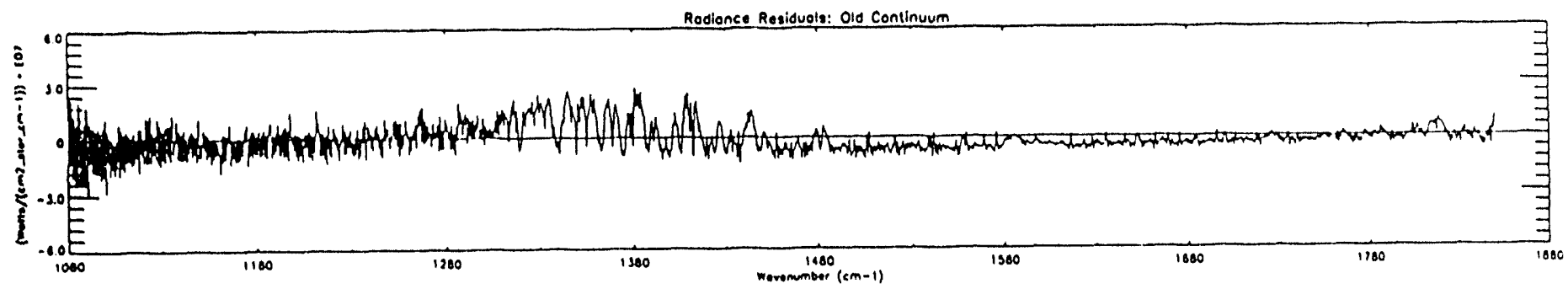

Figure 3b. Spectral residuals from the difference of the observation of figure $3 a$ and an LBLRTM calculation using the CKD continuum.

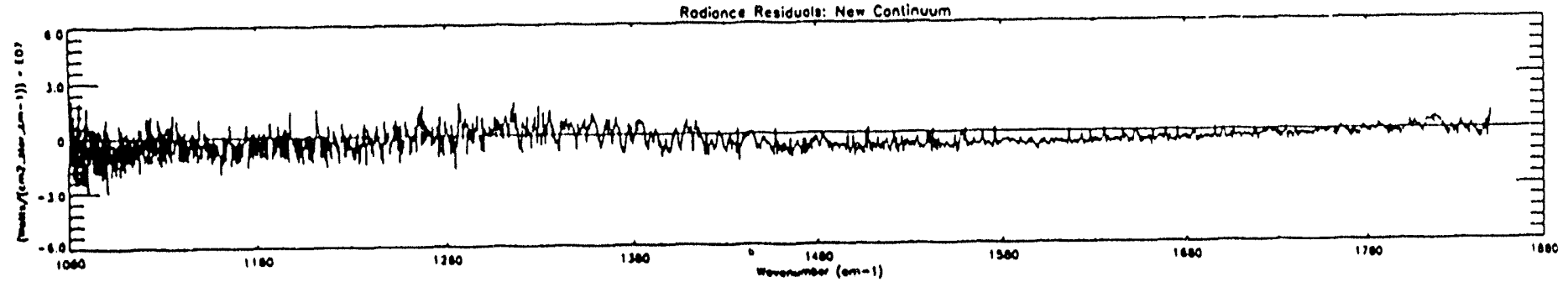

Figure 3c. Spectral residuals from the difference of the observation of figure $3 a$ and an LBLRTM calculation using the CKD-1 continuum. 
continuum does not provide a reduction of these residuals which are most likely attributable to an incorrect methane profile. It should be noted that the upwelling radiance provides an important validation of the treatment of the temperature dependence of the continuum since the radiation is emanating from altitude regimes associated with colder temperatures. The temperature and water vapor profiles have been obtained from radiosonde measurements, the profiles for the uniformly mixed species have been obtained from climatology and the line-by-line calculations are from LBLRTM. Similar conclusions may be drawn from the downwelling radiances from HIS ground based observations. Preliminary results from laboratory measurements by Kulp (private communication, 1993) indicate essential confirmation of the CKD-1 continuum from $700-1300 \mathrm{~cm}^{-1}$ at $296 \mathrm{~K}$.

The CKD water vapor continuum model provides calculated spectra in significantly better agreement with observed spectral radiance data than the Roberts et al. model (1976) which is used in many radiative models for studies related to climate change. Due to its analytical form the Roberts et al. model has a certain appeal related to simplicity of application; nevertheless the model reflects incorrect physics, does not properly treat the foreign coniinuum and is not sufficiently accurate to meet present requirements. The CKD-1 continuum is available to the general scientific community in a stand alone program via anonymous $\mathrm{ftp}$ at AER. The numerical nature of the CKD model makes it readily tractable for application to correlated $\mathrm{k}$-distribution radiative transfer models. The model will continue to undergo validation and refinement. It is anticipated that the spectral radiance data to be obtained at the Western Pacific ARM site will be especially valuable in assessing continuum model performance for tropical atmospheres, a matter of particular importance for climate considerations.

\section{AER}

Progress on the AER Rapid Radiative Transfer Model has been particularly gratifying. This work is being performed by S. Taubman in conjunction with S.A. Clough. R.M. Goody has been an important contributor in a consultive capacity. The study of the k-distribution aspect of the model has been restricted to the $700-825 \mathrm{~cm}^{-1}$ region. This region was shown because it contributes to the cooling rate from the surface through the stratosphere, and involves contributions from both carbon dioxide and water vapor thus testing the correlated-k approach. LBLRTM is used for generating the kdistribution and for providing the reference cooling rate results against which the rapid model is validate. A code, CUMPROB, written by L. Chen of J.P.L. has been provided by M. Gerstell of Cal. Tech. This code develops the cumulative probability distribution from the LBLRTM spectral $k$ values.

Integration schemes have been extensively validated and currently a two stream radiation model for the clear sky is operational. This code has the potential for providing 
highly accurate results with very high computational speed. Fig. 4 indicates the current results for tropospheric fluxes with the mid-latitude summer atmosphere.

\section{Acknowledgements}

We would like to acknowledge the important contributions of several AER scientists including Jean-Luc Moncet, Bob Worsham and Pat Brown. We are also grateful for the many interactions we have had with ARM scientists especially to the $U$. of Wisconsin group under Bill Smith who have been most generous in providing data that have enabled us to achieve improved modeling performance.

\section{References}

Burch, D. E., Continuum absorption by $\mathrm{H}_{2} \mathrm{O}$, Rep. AFGL-TR-81-0300, 32 pp, Air Force Geophys. Lab., Hanscom AFB, MA, 1981.

Burch, D. E., and R. L. Alt, Continuum absorption in the $700-1200 \mathrm{~cm}^{-1}$ and $2400-2800$ $\mathrm{cm}^{-1}$ windows, Rep. AFGL-TR-84-0128, 46 pp., Air Force Geophys. Lab., Hanscom AFB, MA, 1984.

Burch, D. E., Absorption by $\mathrm{H}_{2} \mathrm{O}$ in Narrow Band windows between $3000 \mathrm{~cm}^{-1}$ and $4200 \mathrm{~cm}^{-1}$, Rep. AFGL-TR-85-0036, 38 pp, Air Force Geophys. Lab., Hanscom AFB, MA, 1985.

Clough, S. A., F. X. Kneizys, R.W. Davies, R. Gamache, and R. H. Tipping, Theoretical line shape for $\mathrm{H}_{2} \mathrm{O}$ vapor: application to the continuum, in Atmospheric Water Vapor, edited by A. Deepak, T.D. Wilkerson, and L.H. Rhunke, pp. 25-46, Academic Press, London, 1980.

Clough, S. A., F. X. Kneizys, and R. W. Davies, Line shape and the water vapor continuum, Atmospheric Research, 23, 229-241, 1989.

Clough, S.A., R.D. Worsham, W.L. Smith, H.E. Revercomb, R.O. Knuteson, G. P. Anderson, M.L. Hoke and F.X. Kneizys in IRS '88: Current Problems in Atrinospheric Radiation, edited by J. Lenoble and J. F. Geleyn, pp. 376-379, A. Deepak Publishing. Hampton, VA, 1989.

Clough, S. A., M. J. Iacono and J.-L. Moncet, 1992: Line-by-line Calculations of Atmospheric Fluxes and Cooling Rates: Application to Water Vapor, J. Geophys. Res., 97, 15761-15785.

Ma, Q., and R. H. Tipping, A far wing line shape theory and its application to the water continuum absorption in the infrared region (II), J. Chem. Phys., 96, 8655-8663, 1992.

Roberts, R. E., J. E. A. Selby, and L. M. Biberman, 1976: Infrared continuum absorption by atmospheric water vapor in the $8.12 \mathrm{~mm}$ window, Appl. Opt., 15 , 2085-2090. 
Fluxes derived from LBLRTM and CkD (Correlated k-Distributions)

$$
\begin{gathered}
\text { MLS, CO2, H2O } \\
700-820 \mathrm{~cm}-1
\end{gathered}
$$

Symbols are for CkD derived fluxes (Half gauss: 10 point)

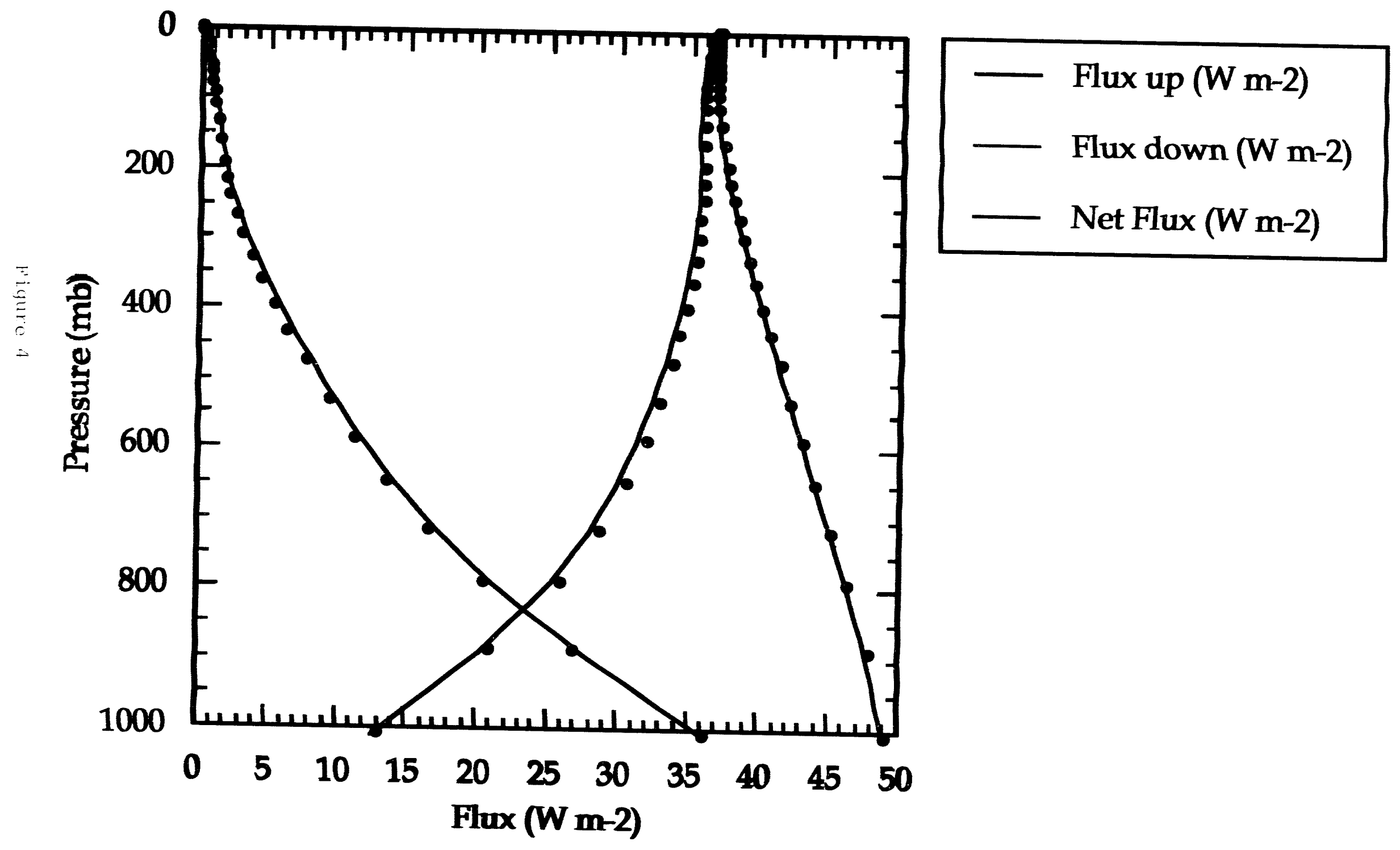


Smith, W. L., H. E. Revercomb, H. B. Howell, and H. M. Woolf, 1983: HIS - A satellite instrument to observe temperature and humidity profiles with vertical resolution, Proc. Fifth Conference on Atmospheric Radiation, AMS.

Toon, O.B., C.P. Mckay, T.P Ackerman and K Santhanam, 1989: Rapid Calculation of Radiative Heating and Photodissociation Rates in Inhomogeneous Multiple Scattering Atmospheres, L. Geophys. Res, 24, 16,287-16,301. 


\title{
RESIDUAL ANALYSIS OF SURFACE SPECTRAL RADIANCES BETWEEN INSTRUMENT OBSERVATIONS AND LINE BY LINE CALCULATIONS
}

\author{
S.A. Clough and P.D. Brown \\ Atmospheric and Environmental Research, Inc. \\ Cambridge, Massachusetts \\ N.E. Miller, J.C. Liljegren and T.R. Shippert \\ Pacific Northwest Laboratories \\ Richland, Washington
}

\section{INTRODUCTION}

The evaluation and improvement of radiative transfer calculations are essential to achieve improved performance of general circulation models (CCM's) for climate change applications. The focus of the study described here is a critical assessment of a radiative transfer experiment conducted under the DoE Atmospheric Radiation Measurement Program (ARM). The three principal components of the study are (1) the ground based measurement of the spectral radiances, (2) the characterization of the atmospheric state associated with the radiating column, and (3) the evaluation of the line by line radiative transfer model. The experiment will be continuous (once per hour) and will be conducted over an extended period of time (up to ten years), important considerations for climate change problems. With respect to global coverage, the first experiment is being conducted in the southern great plains of the United States, with subsequent sites planned for climatologically diverse locations. The initial focus of the experiment is on longwave radiative transfer for clear sky; the approach is to be extended to include the shortwave and atmospheres with clouds and acrosols. The general concept of this type of study is described by Miller et al. (1994).

Evaluation of the present radiation modeling capability is best achieved by using statistical analyses of the residuals between observed and calculated spectral radiances. Spectral radiances are calculated using the Line By Line Radiative Transfer Model (LBLRTM, Clough et al., 1991) in conjunction with the HITRAN92 line parameter database (Rothman et al., 1992). Radiance residuals provide a direct indication of all three components of the experiment by physical process.

Corresponding author address: Shepard A. Clough, Atmospheric and Environmental Rescarch, Inc., 840 Memorial Drive, Cambridgs. MA 02139
In the context of this study, an example of a physical process is the radiance from spectral lines in a specific vibration-rotation band of water vapor. Large spectral residuals associated with water vapor lines could be an indication of problems with the spectroscopy, errors in the observed water vapor profile, errors in the temperature field, or problems with the spectral observations such as the modeling of the instrument function. The methods developed to obtain a quantitative measure of the residuals by physical process are described in this paper. The magnitudes of the means and standard deviations of the residuals provide quantitative measures of the radiative transfer modeling capability over a wide range of atmospheric state. Analyses of these statistical results over time will serve as measures of the capability to observe and model atmospheric radiative transfer and to provide an indication of improvements in that capability.

\section{SPECTRAL RADIANCE OBSERVATIONS}

Radiance spectra are obtained from the University of Wisconsin Atmospheric Emitted Radiance Interferometer (AERI) at the Cloud And Radiation Testbed (CART) facility of ARM. The AERI is a zenith viewing instrument with a spectral resolution of $0.5 \mathrm{~cm}^{-1}$ (unapodized; wavenumber value to first zero). The spectrum is covered in two bands, $520-1800 \mathrm{~cm}^{-1}$ and $1800-3020 \mathrm{~cm}^{-1}$. Radiometric calibrations are done using two high emissivity reference blackbodies (Revercomb et al., 1991). No apodizing function is applied to the data in the interferogram domain other than the rectangular window function, resulting in a sinc scanning function in the spectral domain. The characteristics of the instrument are provided in Table 1. The measured spectral radiances have been corrected to account for the effect of the finite ficld of view. 


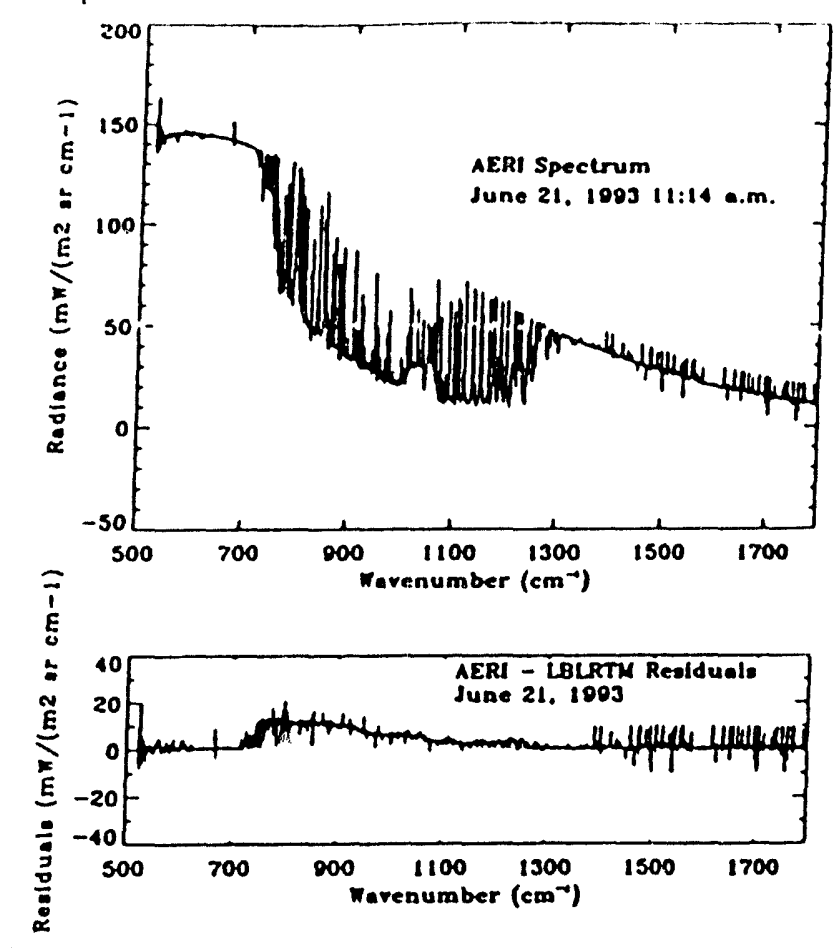

Figure 1. AERI spectrum in the $520-1800 \mathrm{~cm}^{-1}$ region, taken June 21,1993 at 11:14 a.m. (top), and the associated residuals with the LBLRTM calculation (bottom; AERI - LBLRTM).

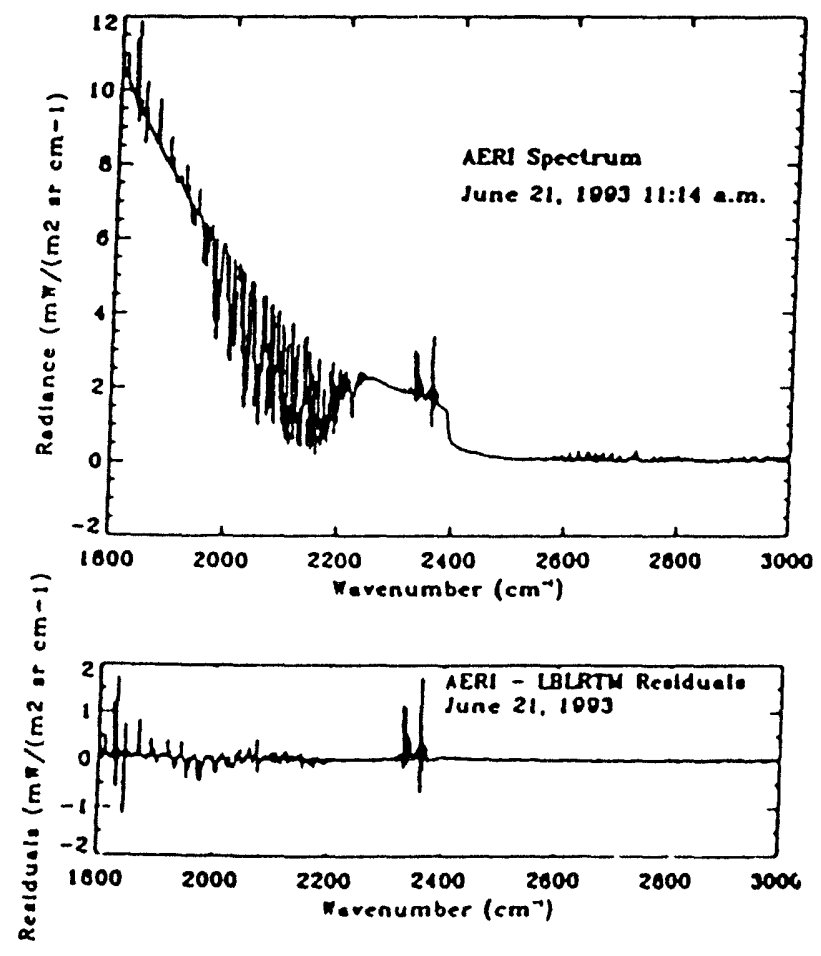

Figure 2. AERI spectrum in the $1800-3000 \mathrm{~cm}^{-1}$ region, taken Junc 21, 1993 at 11:14 a.m. (top). and the associated residuals with LBLRTM calculation (bottom; AERI - LBLRTM).
Figure 1 and Figure 2, are obtained by subtracting the LBLRTM results from the measured spectra. The atmosphere is defined from a radiosonde released at 11:18 a.m., with temperature and water vapor profiles given in Figure 3.

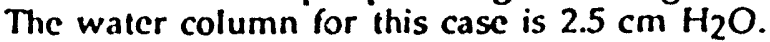
The spectral residuals of Figure 1 correspond to a calculated downwelling flux at the surface approximately $10 \mathrm{w} / \mathrm{m}^{2}$ lower than that measured. The sharp spectral features at $667 \mathrm{~cm}^{-1}$ and from $1400-1800 \mathrm{~cm}^{-1}$ are due to measurement effects associated with strong spectral lines which provide absorption within the instrument and its calibration path.

\section{SPECTRAL MAPPING FUNCTIONS}

A spectral mapping has been developed to associate residuals at each spectral element with a specific physical process. The dependence of spectral elements on molecular species for which the relative density is presumed known correlates elemental sensitivity to the column temperature profile, in addition to the

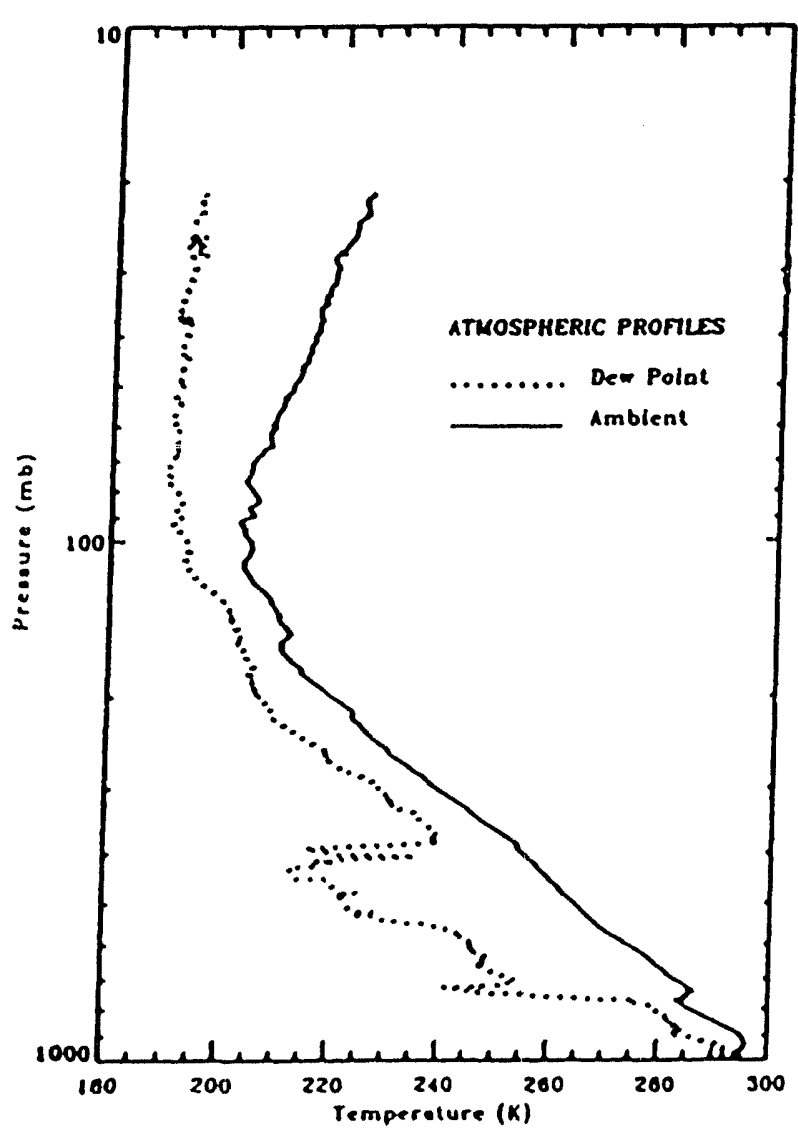

Figure 3. Temperature and water vapor profiles taken from radiosonde launch of June 21, 1993 at $11: 18$ a.m. 
carbon dioxide. It is an unavoidable limitation that the mapping is dependent upon the reference atmosphere chosen, particularly with respect to the variability of water vapor.

The oscillatory bchavior associated with the sinc instrument function introduces ringing into the spectrum at specific spectral elements which may have an adverse effect upon the sensitivity determination and the selection of a dominant species. To assess the effect of this issue, a Gaussian instrument function with the same resolution has been utilized. Results of $Q_{2}$ for both sets of calculations have been compared, and for those spectral elements which are not in agreement the value of 0 , or "indeterminant", has been assigned to the final $Q_{2}$ function. The indeterminant assignment was also made for spectral elements which exhibited strong contributions from more than one physical process. The results of the spectral mapping are shown in Figures $5 \mathrm{a}$ and $5 \mathrm{~b}$.

\section{EXPERIMENT PRODUCTS}

Statistical analyses are performed upon the spectral residuals between the AERI observed radiances and the LBLRTM calculated
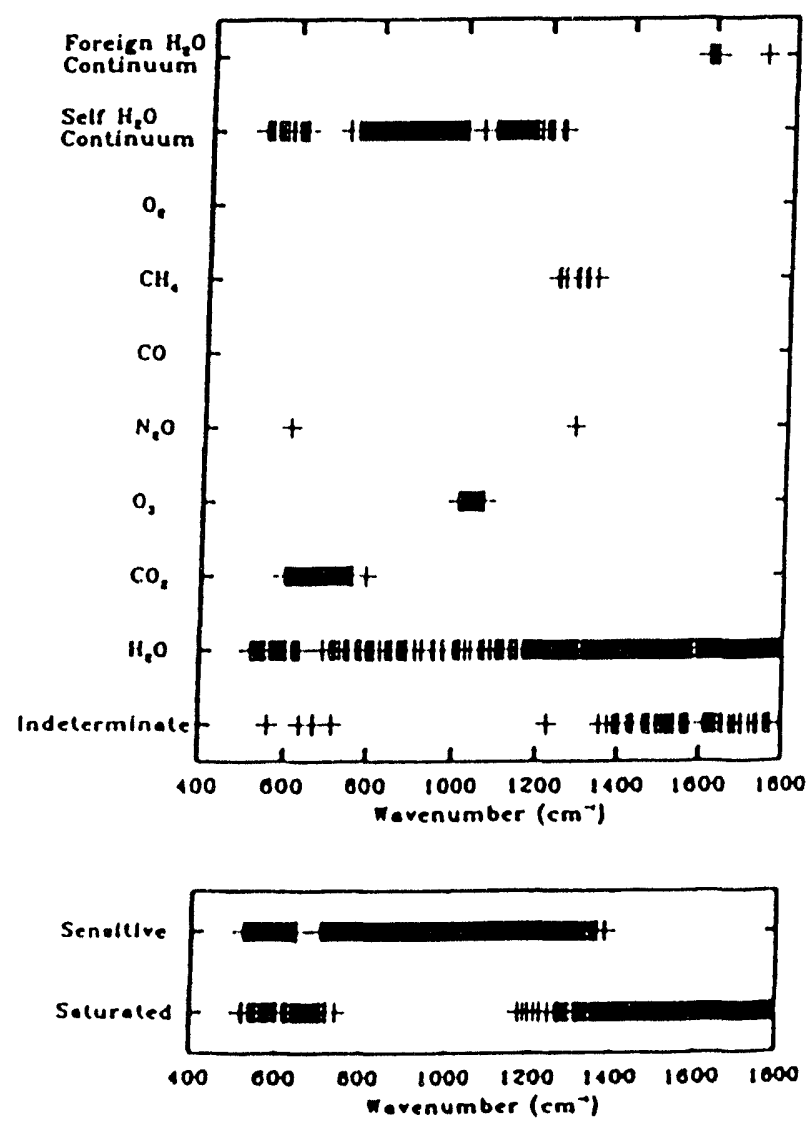

Figure 5a. Final spectral mapping results for the $500-1800 \mathrm{~cm}^{-1}$ region. radiances with regard to the physical process mapping. To facilitate the analysis of the results, seventeen spectral regions demonstrating relatively uniform characteristics with respect to saturation and dominant species have been identified. The criteria used for defining these spectral regions, denoted as "bins," are similar to those used for selecting spectral bands for rapid radiation models used in CCM's. Each bin is defined by a beginning and ending wavenumber, whereby the intersection of any iwo bins is null and the union of all bins is the spectral range of the experiment. Table 2 lists the bins according to spectral interval, sensitivity, and dominant species.

For each bin, the mean and standard deviation about the mean are oblained by process as well as for all processes combined. This approach enables the differentiation of issues associated with water vapor lines, the water vapor continuum, and carbon dioxide lines (indicating potential errors in the temperature profile or modeling of the instrument function) in spectral regions in which all three processes are active (e.g., the $705-799 \mathrm{~cm}^{-1}$ region). In addition, similar statistics are obtained for the broader spectral regions associated with the two
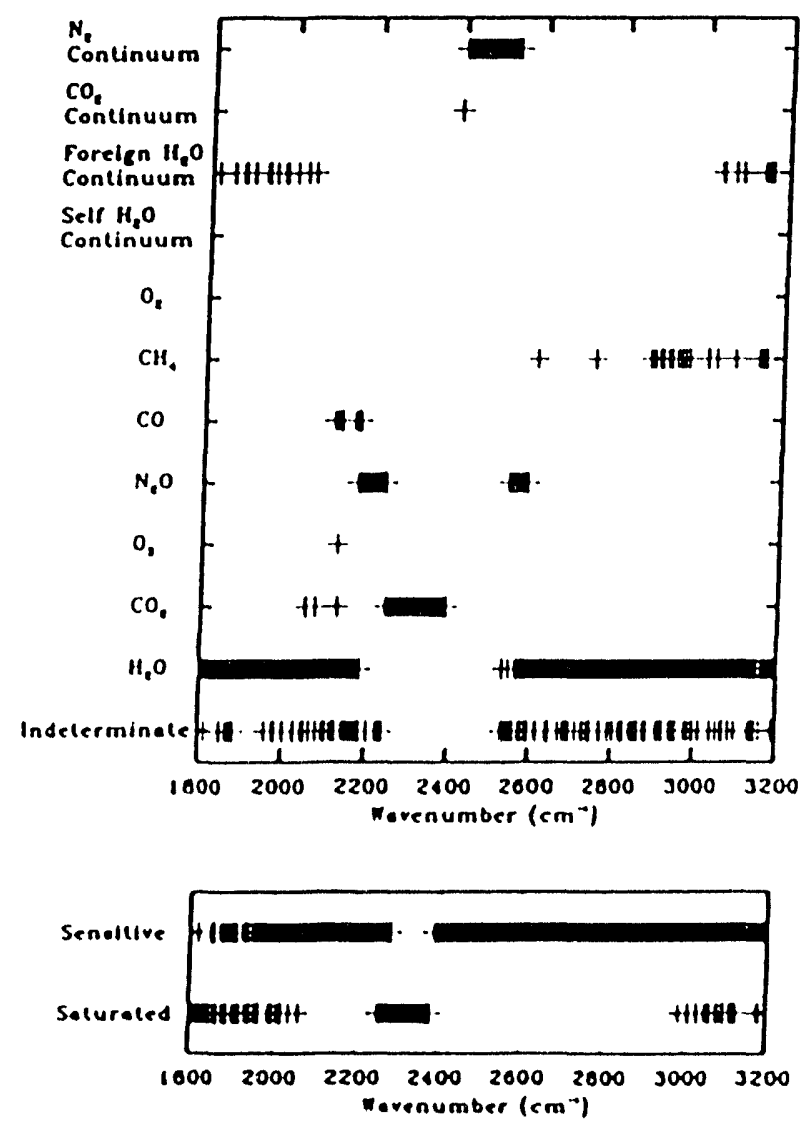

Figure 5b. Final spectral mapping results for the $1800-3200 \mathrm{~cm}^{-1}$ region. 
Clough, S.A., M.J. lacono and J.-L. Moncet, 1992: Line-by-line calculations of atmospheric fluxes and cooling rates: application to water vapor. J. Geophys. Res., 97, 1576115785.

Clough, S.A., J.L. Moncet, R.D. Worsham, M.J. lacono, A. Biancn, 1991: Radiative transfer model development in support of the Atmospheric Radiation Measurement (ARM) Program. Proceedings of the Second Atmospheric Radiation Mcasurement (ARM) Science Team Meeting, 21-24.

Clough, S.A., F.X. Kneizys, and R. W. Davies, 1989: Line shape and the water vapor continuum. Atmospheric Research, 23, 229-241.

Clough, S.A., F.X. Kneizys, E.P. Shettle, and G.P. Anderson, 1985: Atmospheric radiance and transmittance. Proc. of the Sixth Conference on Atmospheric Radiation, 141-144.

Clough, S.A., F.X. Kneizys, L.S. Rothman, and W.O. Gallery, 1981: Atmospheric spectral transmittance and radiance: FASCOD1B. Proc. of Soc. Photo. Opt. Instrum. Eng., 277, 152-166.
Miller, N.E., J.C. Lilgegren, T.R. Shippert, S.A. Clough, and P.D. Brown, 1994: Quality measurement experiments within the Atmospheric Radiation Measurement Program. 74th AMS Annual Mecting, Nashville, Tennessee.

Revercomb, H.E., F.A. Best, R.G. Dedecker, T.P Dirkx, R.A. Herbsleb, R.O. Knuteson, J.F. Short, and W.L. Smith, 1991: High spectral resolution Fourier Transform Infrared (FTIR) instruments for the Atmospheric Radiation Measurement Program: focus on the Atmospheric Emitted Radiance Interferometer. Ploceedings of the Second Atmospheric Radiation Measurement (ARM) Science Team Mecting, 121-124.

Rothman, L.S. , R.R. Gamache, R.H. Tipping, C.P. Rinsland, M.A.H. Smith, D. Chris Benner, V. Malathy Devi, J.-M. Flaud, C. Camy-Peyret, A. Perrin, A. Goldman, S.T. Massie, L.R. Brown and R.A. Toth, 1992: HITRAN molecular database: Edition '92. J. Quant. Spectry. Radiat. Transfer, 48, 469507. 


